Pacific Journal of Mathematic 


\title{
ON LINEAR REPRESENTATIONS OF AFFINE GROUPS I
}

\author{
MANFRED B. WISCHNEWSKY
}

\begin{abstract}
The category of linear representations of an affine group is isomorphic to the category of comodules over a $k$-Hopfalgebra where $k$ denotes a commutative ring. The category of $C$-comodules Comod- $C$ over an arbitrary $k$-coalgebra $C$ is comonadic over the category $k$-Mod of $k$-modules. It is complete, cocomplete and has a cogenerator. The $C$-comodules whose cardinality $\leqq \max \left(\right.$ cardk, $\left.\aleph_{0}\right)$ generate the category Comod- $C$. Comod- $C$ is in general not abelian but can nicely be embedded into an $A B-4$ category. Comod- $C$ is a tensored and cotensored $k$-Mod-category (enriched over $k$-Mod) with a canonical $(E, M)$-factorization which is the factorization in $k$-mod if and only if $C$ is flat. Comod- $C$ has free $C$ comodules if and only if $C$ is finitely generated and projective. Furthermore I give numerous examples and counterexamples as well as the explicit description of all constructions, in particular of the limits in Comod- $C$ which was not known even for coalgebras over fields.
\end{abstract}

Let $k$ be a commutative ring with a unit. $k$-Alg shall denote a small category of models of $k$-algebras (cf. [5] p. XXIV). Recall that an affine $k$-monoid (resp. $k$-group) is a monoid (resp. group) in the functor category [ $k-\mathrm{Alg}$, Sets] whose underlying functor is representable. Let $M$ be a $k$-module. Then $M$ induces an affine $k$-monoid $\mathscr{L}(M): k$-Alg $\rightarrow$ Sets by $\mathscr{L}(M)(A)=\operatorname{End}_{A}\left(M \otimes_{k} A\right), \quad A \epsilon$ $k$-Alg (cf. [5] p. 149). Let $\mathscr{G}$ be an affine $k$-monoid and $M$ a $k$ module. Then a monoid morphism $\varphi: \mathscr{G} \rightarrow \mathscr{L}(M)$ is called a linear representation of $\mathscr{G}$ in $M$ and the pair $(M, \varphi)$ a $k$ - $\mathscr{G}$-module. The definition of morphisms between $k-\mathscr{G}$-modules is evident. Thus one obtains the category $k-\mathscr{G}$-Mod of linear representations of $\mathscr{G}$, resp. of $k$ - $\mathscr{G}$-modules. Since $\mathscr{G}$ is representable we obtain the canonical isomorphisms [k-Alg, Sets] $(\mathscr{G}, \mathscr{L}(M)) \cong \mathscr{L}(M)(C) \cong k$-Mod $\quad(M$, $M \otimes_{k} C$ ), where $C$ is the representing object of $\mathscr{G}$. The monoid structure of $\mathscr{G}$ induces a $k$-coalgebra structure on $C$, i.e., the representing object has two $k$-linear mappings $\Delta: C \rightarrow C \otimes C$ and $\varepsilon: C \rightarrow k$, called comultiplication and counit, such that $\langle C, \Delta, \varepsilon\rangle$ is coassociative and counitary (cf. [19]). By the above canonical isomorphisms every monoid morphism $\varphi: \mathscr{G} \rightarrow \mathscr{L}(M)$ induces a $k$ linear map $\chi_{M}: M \rightarrow M \otimes C$ such that $M \otimes \Delta \cdot \chi_{M}=\chi_{M} \otimes C \cdot \chi_{M}$ and $M \otimes \varepsilon \cdot \chi_{M}=\mathrm{id}_{M}$, and conversely. A pair $\left\langle M, \chi_{M}\right\rangle$ fulfilling the above properties is called a $C$-comodule. Let $\left\langle M, \chi_{M}\right\rangle$ and $\left\langle N, \chi_{N}\right\rangle$ be $C$ comodules. $A k$-linear mapping $f: M \rightarrow N$ is a $C$-comodule homo- 
morphism if $\chi_{N} \cdot f=f \otimes C \chi_{M}$. Let $\left(M, \varphi_{M}\right)$ and $\left(N, \varphi_{N}\right)$ be $k-\mathscr{G}$ modules and $\left\langle M, \chi_{M}\right\rangle$, resp. $\left\langle N, \chi_{N}\right\rangle$ the corresponding $C$-comodules. Then a $k$-linear mapping $f: M \rightarrow N$ is a $k$ - $\mathscr{G}$-module homomorphism $f:\left(M, \varphi_{M}\right) \rightarrow\left(N, \varphi_{N}\right)$ if and only if $f:\left\langle M, \chi_{M}\right\rangle \rightarrow\left\langle N, \chi_{N}\right\rangle$ is a $C$ comodule homomorphism.

Hence the category of linear representations of an affine monoid (group) is isomorphic to a category of $C$-comodules where $C$ is a $k$-bialgebra (resp. $k$-Hopf algebra).

In this paper I study the elementary properties of a category of comodules over an arbitrary $k$-coalgebra. Categories of comodules were already studied by several authors where $k$ is a field or the coalgebra is finite or flat (cf. [5], [7], [10], [14], [15], [17], [18], [19]). In all these cases Comod- $C$ is a Grothendieck category with a generator. But if $C$ is not flat then Comod- $C$ need not to be abelian. This was already shown in [17]. The homomorphism theorem is no longer valid, the comodule structure on a subcomodule is in general no longer unique and so on.

But even in the case of a flat coalgebra $C$ one didn't know as yet such elementary things as the explicit descriptions of limits.

Let $C$ be an arbitrary coalgebra over a commutative ring $k$ with a unit. Then the most important results of this paper are: The underlying functor $U$ : Comod- $C \rightarrow k$-Mod is comonadic. The category Comod- $C$ is complete, cocomplete, wellpowered and cowellpowered, has a generator and cogenerator. Comod- $C$ can be embedded (full and faithful) into an $A B 4$-category with sufficiently many injectives and projectives which in general fails to be a Grothendieck-category. This embedding is coreflective if and only if all objects in Comod-C are projective and is an isomorphism if and only if Comod- $C$ is a spectral category. The functor $\lambda$ : Comod-C $\rightarrow C^{*}-\operatorname{Mod}$ (cf. [14] $\S 1$ or [19] Chap. II) is comonadic. Comod- $C$ has free comodules if and only if $C$ is finitely generated and projective. Comod- $C$ has a proper ( $E, M$ )-factorization which is preserved by the underlying functor Comod- $C \rightarrow k$-Mod if and only if $C$ is flat. Comod- $C$ is well-powered and cowellpowered with respect to this factorization. By applying the techniques of $V$-categories I show that the $k$-Mod-category Comod- $C$ is tensored and cotensored. If $f: C \rightarrow C^{\prime}$ is coalgebra morphism then the induced $k$-linear functor $f^{*}:$ Comod- $C \rightarrow \operatorname{Comod}-C^{\prime}$ preserves tensors and is $k$-Mod-comonadic. The $k$-linear functor $-\otimes C: k$-Mod $\rightarrow$ Comod- $C$ has a $k$-linear-right adjoint. Furthermore I give numerous examples and counterexamples as well as explicit descriptions of all constructions.

I. Comodules over arbitrary coalgebras. In the language of 
monoidal categories a $k$-coalgebra $\langle C, \Delta, \varepsilon\rangle$ is just a comonoid in the monoidal category ( $k$-Mod, $\otimes$ ) (cf. [11] Chap. VII 3). A $C$-comodule $\left\langle M, \chi_{M}\right\rangle$ is a coaction of $C$ on $M$ and a $C$-comodule homomorphism is a morphism between coactions of $C$ in $(k-\bmod , \otimes)$ (cf. [11] Chap. VII 4). This formal description gives us at once some elementary results such as the existence of a right adjoint of the underlying functor $U$ : Comod- $C \rightarrow k$-Mod or the creation of colimits by $U$.

In the sequel I will give another description of Comod- $C$ which allows us to apply the highly developed theory of monads.

Let $\langle C, \Delta, \varepsilon\rangle$ be a coalgebra. The coalgebra structure of $\langle C, \Delta, \varepsilon\rangle$ induces a functor

$$
\mathscr{C}:=-\otimes C ; k-\operatorname{Mod} \longrightarrow k-\operatorname{Mod}
$$

and functorial morphisms

$$
\begin{aligned}
& \Delta=-\otimes \Delta: \mathscr{C} \longrightarrow \mathscr{C}^{2}=-\otimes C \otimes C \\
& \varepsilon=-\otimes \varepsilon: \mathscr{C} \longrightarrow I d_{k-\mathrm{Mod}} .
\end{aligned}
$$

Since $\langle\mathrm{C}, \Delta, \varepsilon\rangle$ is a coalgebra $\langle-\otimes C,-\otimes \Delta,-\otimes \varepsilon\rangle$ clearly defines a comonad over $k$-Mod. A coalgebra $\left\langle M, \chi_{M}\right\rangle$ over this comonad is a pair where $M$ is $k$-module and $\chi_{M}: M \rightarrow \mathscr{C}(M)$ is a $k$-morphism such that the following diagrams commutes

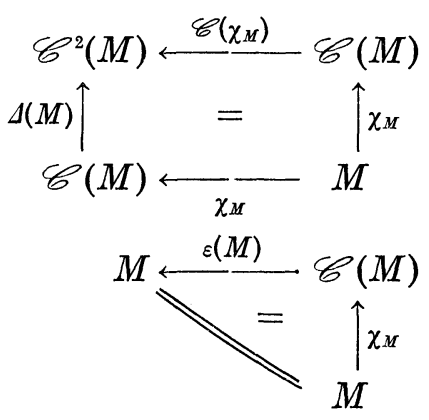

A morphism $f$ between $\mathscr{C}$-coalgebras $\left\langle M, \chi_{M}\right\rangle$ and $\left\langle N, \chi_{N}\right\rangle$ is a $k$-morphism $f: M \rightarrow N$ such that $\chi_{N} \cdot f=\mathscr{C}(f) \cdot \chi_{M}$. Hence we obtain the following

Theorem 1 (Notation as above). Let $\langle C, \Delta, \varepsilon\rangle$ be a coalgebra. Then the category Comod-C of C-comodules is comonadic over $k$-Mod.

From the elementary theory of monads we obtain at once some important corollaries. 


$$
U: \text { Comod- } C \longrightarrow k-\mathrm{Mod}
$$

has a right adjoint $\mathscr{G}: k$-Mod $\rightarrow$ Comod- $C$ defined by

$$
\begin{aligned}
\mathscr{G}: k-\operatorname{Mod} & \longrightarrow \text { Comod- } C \\
M & \longmapsto\langle M \otimes C, M \otimes \Delta\rangle \\
f & \longmapsto f \otimes C
\end{aligned}
$$

The comonad defined in k-Mod by this adjunction is the given comonad $\langle-\otimes C,-\otimes \Delta,-\otimes \varepsilon\rangle$.

COROLLARY 3. The underlying functor $U$ : Comod- $C \rightarrow k$-Mod creates colimits and isomorphisms. In particular Comod-C is cocomplete and the colimits are formed in k-Mod.

COROLLARY 4. U creates those limits which are preserved by - $\otimes C$. If $C$ is flat and $T: D \rightarrow$ Comod-C is a finite diagram, then $p$ : Diag $M \rightarrow T$ is a limit in Comod-C if and only if Up: Diag $U M \rightarrow U T$ is a limit in k-Mod.

Applying 21.3.6 in [16] we obtain

Corollary 5. Comod-C is cowellpowered.

Since right adjoints preserve cogenerators we get

Corollary 6. Comod-C has a cogenerator.

Let $\mathscr{C}$ be a category with finite limits and finite colimits. A functor $F: C \rightarrow C^{\prime}$ is called left-exact (right-exact) if $F$ preserves finite limits (finite colimits). $F$ is called exact if $F$ is left-exact and right-exact.

Since $k$-Mod is an additive category and $-\otimes C$ is additive and right-exact we obtain from Remark 21.1.11 in [16] Chap. 21 the well known

CoROllary (cf. [7], [10]).

(1) Comod- $C$ is an additive category.

(2) $U$ and $\mathscr{G}$ are additive functors.

Furthermore $\mathscr{G}$ is exact and $U$ is right exact.

Proposition 8 (Notation as above). The following statements are equivalent:

(i) $U$ is exact. 
(ii) $C$ is flat.

(iii) $\mathscr{G}$ preserves injectives.

Proof. (ii) $\rightarrow$ (i): Since $U$ creates finite limits and is right exact it is exact.

(i) $\rightarrow$ (ii): Let $f: M \rightarrow N$ be an injective $k$-module homomorphism. Since $\mathscr{G}$ is exact, $\mathscr{G}(f)=f \otimes C: M \otimes C \rightarrow N \otimes C$ is an equalizer in Comod-C. Since $U$ is exact $f \otimes C$ is injective, i.e., $C$ is flat.

(i) $\rightarrow$ (iii): Well known.

(iii) $\rightarrow$ (i): Let $m:\left\langle M, \chi_{M}\right\rangle \rightarrow\left\langle N, \chi_{N}\right\rangle$ be a monomorphism in Comod- $C$ and $f: M \rightarrow Q$ an injective extension of $M$ in $k$-Mod. Then we obtain the following commutative diagram

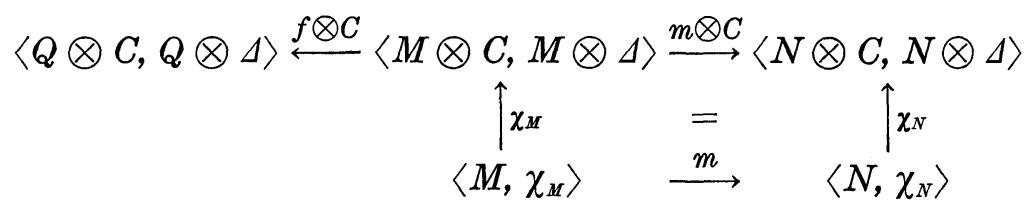

Since $\mathscr{G}$ preserves injectives, $\langle Q \otimes C, Q \otimes \Delta\rangle=\mathscr{G}(Q)$ is injective in Comod-C. Since $\mathscr{G}(Q)$ is injective and $m$ is a monomorphism we obtain a comodule-homomorphism $g:\left\langle N, \chi_{N}\right\rangle \rightarrow\langle Q \otimes C, Q \otimes \Delta\rangle$ such that

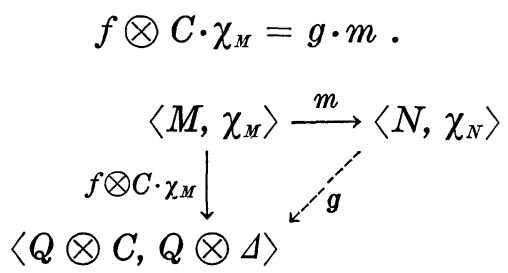

Since $\left\langle M, \chi_{\mu}\right\rangle$ is a $C$-comodule and $\varepsilon:-\otimes C \rightarrow I d_{k-\text { Mod }}$ is a functorial morphism we obtain the following equations:

$$
\varepsilon_{M} \cdot \chi_{M}=i d_{M} \text { and } f \cdot \varepsilon_{M}=\varepsilon_{Q} f \otimes C \text {. }
$$

Thus $f=f \cdot \mathrm{id}_{M}=f \cdot \varepsilon_{M} \cdot \chi_{M}=\varepsilon_{Q} \cdot f \otimes C \chi_{M}=\varepsilon_{Q} \cdot g \cdot m$. Hence $m$ is injective since $f$ is injective, i.e., $U$ is exact.

If $C$ is flat $U$ creates finite limits and colimits. Since Comod-C is additive and $k$-Mod is abelian we conclude that Comod- $C$ is abelian. Since furthermore $k$-Mod is a Grothendieck category and $U$ preserves and reflects colimits and monomorphisms Comod- $C$ fulfills $A B 5^{\prime}$ (cf. [16] 4,6.3), i.e., we obtain the following well known result.

Corollary 9. If $C$ is flat then Comod-C is a Grothendieck category. Furthermore $U$ preserves and reflects finite limits and 
colimits. In particular a comodule homomorphism is an equalizer (coequalizer) in Comod- $C$ if and only if $f$ is injective (surjective).

EXAMPLES 10. (1) Let $\mathrm{k}$ be a regular ring (regular in the sense of von Neumann) (cf. [2] p. 175, EX. 13). Then Comod-C is a Grothendieck category for every $k$-coalgebra $C$.

Let $k$ be a commutative, associative ring with unit. Let $T$ be a $k$-module. Then $C=k \oplus T$ together $\Delta(r, t)=r \otimes 1+1 \otimes t+$ $t \otimes 1+\rho(t)$ and $\varepsilon(r, t)=r$ is a coalgebra with unit (cf. [18], where $\rho: T \rightarrow T \otimes T$ is an arbitrary coassociative $k$-morphism (take for example $\rho=0$ ). Hence $C=k \oplus T$ is flat (projective, finitely generated, ...) if and only if $T$ is flat (projective, finitely generated, ...).

(2) Let $A$ be a torsion free abelian group $A$ and $C=Z \bigoplus A$ with the above defined structure ${ }^{\cdot}$ Then Comod- $C$ is a Grothendieck category ${ }^{1}$.

(3) Let $A$ be an abelian group which is not torsion free. (e.g., $\boldsymbol{Z} / n \boldsymbol{Z}, \boldsymbol{Q} / \boldsymbol{Z})$. Then the coalgebra $C=\boldsymbol{Z} \oplus A$ with one of the above defined coalgebra structures is not flat ${ }^{1}$.

Definition 11. Let $\left\langle M, \chi_{M}\right\rangle$ be a $C$-comodule. A subcomodule $\left\langle N, \chi_{N}\right\rangle$ is a submodule $N$ of $M$ such that the inclusion $i: N \rightarrow M$ is a comodule homomorphism.

Proposition 12. Let Comod-C be an abelian category. Then the comodule structure on a subcomodule is unique.

Proof. Let $\left\langle N, \chi_{1}\right\rangle$ and $\left\langle N, \chi_{2}\right\rangle$ be subcomodules of $\left\langle M, \chi_{M}\right\rangle$. Since the inclusion $i:\left\langle N, \chi_{1}\right\rangle \rightarrow\left\langle M, \chi_{M}\right\rangle$ is injective it is a monomorphism and hence an equalizer in Comod- $C$ since Comod- $C$ is abelian by assumption. Hence the identity $\left\langle N, \chi_{2}\right\rangle \rightarrow\left\langle N, \chi_{1}\right\rangle$ must be a comodule homomorphism. Since $U$ : Comod- $C \rightarrow k$-Mod creates isomorphisms we obtain $\chi_{1}=\chi_{2}$.

EXAMPLE 13. (cf. [18]) Let $C=\left.\boldsymbol{Z} \oplus \boldsymbol{Z}\right|_{n \boldsymbol{Z}}$ be the $Z$-coalgebra with the following structure:

$$
\begin{aligned}
& \Delta(z, \bar{q})=z \otimes 1+1 \otimes \bar{q}+\bar{q} \otimes 1+\bar{q} \otimes \overline{1} \\
& \varepsilon(z, \bar{q})=z . \text { (cp. (11) Ex. 1) }
\end{aligned}
$$

Then the category Comod- $C$ of $\boldsymbol{Z} \oplus \boldsymbol{Z} / n \boldsymbol{Z}$-comodules is not abelian. By applying Proposition 12 we have only to show that there exist a $C$-comodule $\left\langle M, \chi_{M}\right\rangle$ and subcomodules $\left\langle N, \chi_{N}\right\rangle$ and $\left\langle N, \chi_{N}^{\prime}\right\rangle$ of

${ }^{1}$ Let $k$ be a principal ideal domain. Then a $k$-module $M$ is flat if and only if $M$ is torsion free (cf. [4] §24 Prop. 3 (ii)). 
$\left\langle M, \chi_{M}\right\rangle$ with $\chi_{N} \neq \chi_{N}^{\prime}$. The following example was given in [18]. Take

$$
\begin{aligned}
& M=\boldsymbol{Q} / \boldsymbol{Z} ; \chi_{M}(\bar{q})=\bar{q} \otimes 1 \\
& N=\boldsymbol{Z} / n \boldsymbol{Z} ; \chi_{N}(\bar{z})=\bar{z} \otimes 1
\end{aligned}
$$

and

$$
N=Z / n Z ; \chi_{N}^{\prime}(\bar{z})=\bar{z} \otimes 1+\overline{1} \otimes \bar{z} .
$$

Then the inclusion $i: \boldsymbol{Z} / n \boldsymbol{Z} \rightarrow \boldsymbol{Q} / \boldsymbol{Z}: \bar{z} \rightarrow(\bar{z} / n)$ is a comodule homomorphism for $\chi_{N}$ and $\chi_{N}^{\prime}$. Since $\chi_{N} \neq \chi_{N}^{\prime}$ we obtian that Comod-C is not abelian.

Conjecture 14. Comod-C is abelian if and only if $C$ is flat.

In order to prove this conjecture one has to show that if Comod- $C$ is abelian then the comodule monomorphisms are injective (cf. Proposition 8).

In [9], P. Freyd proves the existence of free abelian categories. $\mathrm{He}$ does it by taking a category $C$ and embedding it into a large ambient abelian category. He then constructs the smallest exact subcategory containing $C$. The external version of this construction was made by M. Alderman in [1]. He gives an explicit description of free abelian categories. I'll take up Alderman's construction and will show that the category Comod- $C$ (for every coalgebra $C$ ) can be fully and faithfully embedded into an $A B-4$ category with enough projectives and injectives, the free abelian category over Comod-C which in general fails to be a Grothendieck category.

Let us now recall Alderman's construction. Let $\boldsymbol{A}$ be an additive category. In the functor category $\vec{A} \rightarrow$ define the following equivalence relation:

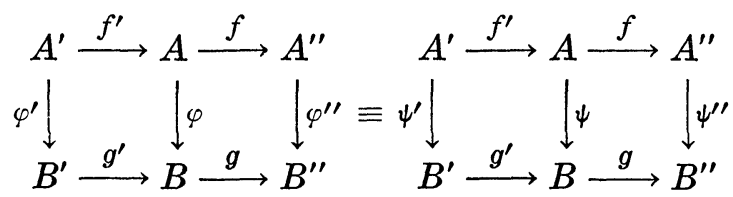

iff there are maps $h_{1}: A \rightarrow B^{\prime}$ and $h_{2}: A^{\prime \prime} \rightarrow B$ such that $\varphi-\psi=$ $g^{\prime} h_{1}+h_{2} f$, i.e., the two short complexes are homotopic. Then the resulting category $\boldsymbol{A} \rightarrow \mid \equiv$ is denoted by $A b(\boldsymbol{A}) . \quad A b(\boldsymbol{A})$ is abelian ([1]). The functor $I_{A}: A \rightarrow A b(A): A \rightarrow(0 \rightarrow A \rightarrow 0)$ is obviously full and faithful. Let now $F$ be an additive functor from $\boldsymbol{A}$ to $B$ with $\boldsymbol{B}$ abelian. Then there is a unique exact functor $F^{*}: A b(\boldsymbol{A}) \rightarrow \boldsymbol{B}$ such that the diagram 


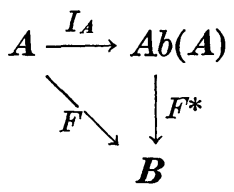

commutes up to natural equivalence (cf. [1] Theorem 1.14).

Let now $\boldsymbol{A}$ be the additive category Comod- $C$.

THeorem 15. Let $C$ be a coalgebra. Then

(1) There exists an abelian category $A b$ (Comod-C) and a full and faithful embedding

$$
I: \text { Comod- } C \longrightarrow A b \text { (Comod- } C)
$$

such that every additive functor $F$ : Comod- $C \rightarrow B$ into an abelian category $B$ can be factored through an exact functor $F^{*}: A b$ (Comod-C) $\rightarrow$ $B$ (up to natural equivalence).

(2) $A b$ (Comod-C), the free abelian category over Comod- $C$, is an AB4-category.

(3) The inclusion functor I preserves products and coproducts.

(4) The inclusion functor I preserves equalizers (coequalizers) if and only if the equalizers (coequalizers) in Comod-C are coretractions (retractions).

(5) $A b$ (Comod-C) has sufficiently many projectives and injectives.

As immediate consequences of this theorem we obtain the following two theorems by applying the special adjoint functor theorem:

THEOREM 16 (Notation as above). The following statements are equivalent.

(i) Comod-C is a coreflective subcategory of $A b$ (Comod- $C$ ).

(ii) The inclusion functor $I$ : Comod- $C \rightarrow A b$ (Comod-C) preserves epimorphisms.

(iii) Every epimorphism in Comod-C is a retraction.

(iv) Every object in Comod-C is projective.

THEOREM 17 (Notation as above). The following statements are equivalent:

(i) The inclusion $I$ : Comod-C $\rightarrow A b($ Comod-C) is an isomorphism.

(ii) Every object in Comod-C is injective.

(iii) Every monomorphism in Comod-C is a coretraction. If (i)-(iii) are fulfilled then Comod-C is a spectral category.

REMARK 18. If Comod-C is an abelian category then the 
statements of the above two theorems are equivalent. But if Comod- $C$ is not abelian then these conditions need not to be equivalent.

Proof of Theorem 15. We have to prove (2), (3), (4) since the other statements were proved in [1].

(2) Let $M_{i}^{\prime} \stackrel{f^{\prime} i}{\longrightarrow} M_{i} \stackrel{f^{\prime} i}{\longrightarrow} M_{i}^{\prime \prime}, i \in I$, be a family of $A b$ (Comod- $C$ )objects. Then

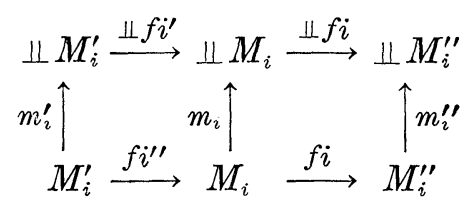

is the coproduct of these family in $A b$ (Comod-C) as one easily shows, where $m_{i}^{\prime}, m_{i}$ and $m_{i}^{\prime \prime}, i \in I$ are the corresponding coproducts of the objects $M_{i}^{\prime}, M_{i}$ and $M_{i}^{\prime \prime}$ in Comod-C. Hence $A b$ (Comod-C) is cocomplete, i.e., an $A B-3$ category. In order to show that $A b$ (Comod-C) is an $A B 4$-category we have to show that for any family $\left\{f_{i}:\left(M_{i}\right) \rightarrow\left(N_{i}\right)\right\}$ of monomorphisms in $A b$ (Comod-C), the morphism $\Perp f_{i}$ is also a monomorphism.

LEMma 19 ([1] Theorem 1.1 or [8] Lemma 6.1).

(1) The equalizer of

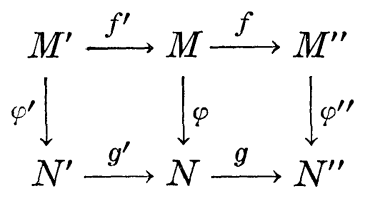

is given by

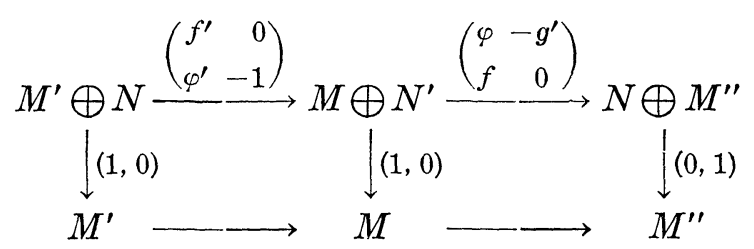

and the coequalizer by

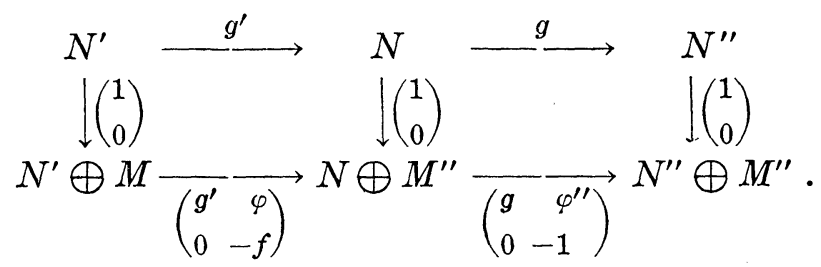


Since $A b($ Comod-C) is an abelian category we obtain at once the following criterium.

LEMMA 20. Let

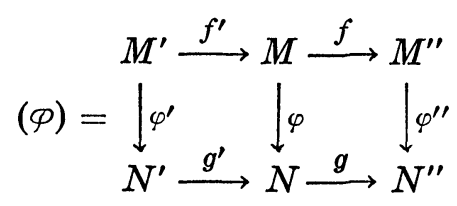

be a morphism in $A b$ (Comod-C). Then

(1) ( $\varphi$ ) is a monomorphism if and only if there are morphisms

$$
\begin{aligned}
\psi^{\prime}: N^{\prime} \longrightarrow M^{\prime}, q: M \longrightarrow M^{\prime} & \longrightarrow \text { and } \psi: N \longrightarrow M \text { such that } \\
q^{\prime \prime}: M^{\prime \prime} \longrightarrow & \longrightarrow M \text { and } \\
& f^{\prime} q+\psi \cdot \varphi+q^{\prime \prime} \cdot f=\mathrm{id}_{M}
\end{aligned}
$$

and

$$
f^{\prime} \cdot \psi^{\prime}+\psi \cdot g^{\prime}=0
$$

(2) ( $\varphi$ ) is an epimorphism if and only if there are morphisms

$$
\begin{aligned}
p: N \longrightarrow & N^{\prime}, p^{\prime \prime}: N^{\prime \prime} \longrightarrow N, \\
\delta: N \longrightarrow & M \text { and } \delta: N^{\prime \prime} \longrightarrow M^{\prime \prime} \text { such that } \\
& g^{\prime} \cdot p+p^{\prime \prime} g+\varphi \cdot \delta=\mathrm{id}_{N} \\
& \delta^{\prime \prime} g+f \cdot \delta=0 .
\end{aligned}
$$

The construction of coproducts in $A b$ (Comod-C) and Lemma (20) 1 show immediately that $A b$ (Comod- $C$ ) is an $A B 4$-category.

(3) Trivial.

(4) Let $f: M \rightarrow N$ an equalizer in Comod-C and assume that I preserves this equalizer

Consider the following diagram

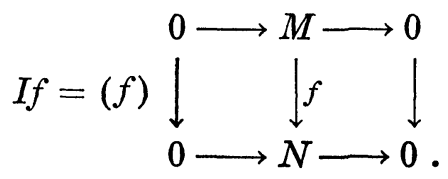

Then $(f)$ is a monomorphism in $A b$ (Comod- $C$ ) if and only if there exists a morphism $g: N \rightarrow M$ such that $g \cdot f=\mathrm{id}_{m}$, i.e., if $f$ is a coretraction (Lemma 20.1). In the same vein one shows by applying Lemma 20.2 that $f$ is an epimorphism if and only if $f$ is a retraction Comod-C. This completes our proof. 
REMARK 21. (1) $A b$ (Comod-C) is an $A B 4^{*}$-Category. Let $C$ be a coalgebra. Then Comod- $C$ is complete by Corollary 26. Now in the same vein as above one shows that $A b$ (Comod-C) has products which are the pointwise ones. Hence $A b$ (Comod- $C$ ) is an $A B 3^{*}$ category. From the construction of products and the characterization of epimorphisms by Lemma 20.2 we obtain that $A b$ (Comod-C) is an $A B 4^{*}$-category.

(2) $A b$ (Comod-C) is, in general, not a Grothendieck category. Take $\boldsymbol{Z}$ with the trivial coalgebra structure. Then Comod- $\boldsymbol{Z}$ is isomorphic to $Z$-Mod, the category of abelian groups. Assume $A b($ Comod- $Z)=A b(Z$-Mod $)$ is a Grothendieck category. Since $A b$ ( $Z$-Mod) is an $A B 3^{*}$-category by $211, A b\left(Z\right.$-Mod) is a $C_{2}$-category (Mitchell [12]), i.e., for any set $\left(M_{i}\right)$ of objects in $A b$ (Z-Mod) the canonical morphism

$$
m: \Perp M_{i} \longrightarrow \pi M_{i}
$$

is a monomorphism. Take now $M_{n}=Z$ for $n \in N$. Then the canonical morphism

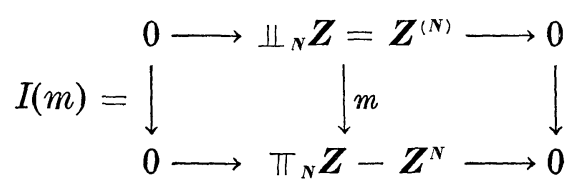

is the image of the canonical morphism $m: Z^{(N)} \rightarrow Z^{N}$. Then $I(m)$ is a monomorphism in $A b(Z$-Mod) if and only if the canonical morphism $m: \boldsymbol{Z}^{(\boldsymbol{N})} \rightarrow \boldsymbol{Z}^{N}$ is a coretraction. Consider now the canonical projection $p: \boldsymbol{Z}^{N} \rightarrow \boldsymbol{Z}^{N} / \boldsymbol{Z}^{(N)}$ and the element $\bar{x}=\left(2^{n} ; n \in N\right) \in \boldsymbol{Z}^{N}$. Then the image $p(\bar{x})$ is obviously divisible by every power of 2 . Since an element $\left(x_{i} ; i \in I\right)$ in $\boldsymbol{Z}^{I}$ is divisible if and only if all components $x$ are divisible in $\boldsymbol{Z}$ we obtain that $\boldsymbol{Z}_{N} \mid \boldsymbol{Z}^{(N)}$ cannot be embedded in a product $\boldsymbol{Z}^{I}$. Hence the monomorphism $m: 0 \rightarrow \boldsymbol{Z}^{(N)} \rightarrow \boldsymbol{Z}^{N}$ is not split, i.e., no coretraction and therefore $I(f)$ is no monomorphism in $A b$ ( $Z$-Mod). Hence $A b$ (Comod- $Z$ ) is not a Grothendieck category.

Next I will prove that Comod- $C$ has a generator where $C$ is an arbitrary coalgebra. The existence of a generator in Comod- $C$ where $C$ is flat was proved by Saavedra [15] 2.07. But his proof cannot be generalized. The following proof uses Barr's results in [3[ and is in fact an imitation of his proof of the existence of a set of generators in the category of coalgebras over a commutative ring.

A submodule $U \subset M$ of a module $M$ is called a pure submodule of $M$ provided that for any module $N U \otimes N \rightarrow M \otimes N$ is a monomorphism.

Proposition 22 (Barr [3] 1.3). Given $U \subset M$ there is an $U^{*} \subset M$ 
such that $U \subset U^{*}$ such that $U^{*}$ is a pure submodule of $M$, and such that

$$
\operatorname{card}\left(U^{*}\right) \leqq \max \left(\operatorname{card}(U), \operatorname{card}(k), \chi_{0}\right) .^{2}
$$

THEOREM 23. Let $\langle M, \chi\rangle$ be a C-comodule, $U$ a submodule of $M$. Then there is a subcomodule $M^{\prime} \subset M$ such that $U \subset M^{\prime}$ and

$$
\operatorname{card}\left(M^{\prime}\right) \leqq \max \left(\operatorname{card} U, \operatorname{card} k, \aleph_{0}\right) \text {. }
$$

Proof. Let $\langle M, \chi\rangle$ be a $C$-comodule. A $k$-submodule $U$ of $M$ is called $\chi$-invariant if $\chi(U) \subset i \otimes C(U \otimes C)$ where $i: U \rightarrow M$ is the inclusions. Let $U$ be a submodule of $M$. For each $u \in U$ choose a representation

$$
\chi(u)=\sum_{i=1}^{n} m_{i} \otimes C_{i} .
$$

Let $U^{\prime}$ be the submodule generated by all $m_{i}$ and the elements of $U$. Then $U \subset U^{\prime} \subset M, \quad \chi(U)=\sum_{i=1}^{n} m_{i} \otimes C_{i} \in i \otimes C\left(U^{\prime} \otimes C\right)$ and $\operatorname{card}\left(U^{\prime}\right) \leqq \max \left(\operatorname{card} U\right.$, $\left.\operatorname{card} k, \chi_{0}\right)$.

Now iterate the above process in order to get a sequence

$$
U \subset U^{\prime} \subset U^{\prime \prime} \subset \cdots \subset U^{(n)} \subset \cdots
$$

such that $\chi\left(U^{(n)}\right) \subset i \otimes C\left(U^{(n+1)} \otimes C\right)$. Define $\hat{U}=\bigcup_{n \in N} U^{(n)}$. Then $\hat{U}$ is a submodule of $M$ such that $U \subset \hat{U}$ such that $\hat{U}$ is $\chi$-invariant and such that $\operatorname{card}(\hat{U}) \leqq \max \left(\operatorname{card} U\right.$, card $\left.k, \chi_{0}\right)$. Next we define the following sequence of submodule of $M$

$$
U_{n}=U_{n-1}^{*} \quad \text { when } n \text { is odd }
$$

and

$$
U_{n}=\hat{U}_{n-1} \text { when } n \text { is even, }
$$

where $U_{n-1}^{*}$ is "the" pure submodule of $M$ containing $U_{n-1}(\rightarrow$ Proposition 22). Then let $M^{\prime}=\bigcup U_{n}$. Then $M^{\prime} \subset M$ is a pure submodule of $M$ which is $\chi$-invariant. Hence $\chi\left(M^{\prime}\right) \subset M^{\prime} \otimes C$ and $\left\langle M^{\prime}, \chi\right\rangle$ is a subcomodule of $\langle M, \chi\rangle$. The cardinality conclusion is obvious.

THEOREM 24. The $C$-comodule whose cardinality $\leqq \max \left(\operatorname{card} k, \boldsymbol{\aleph}_{0}\right)$ generate the category Comod-C. In particular Comod-C has a generator.

Proof. Let $f, g:\left\langle M, \chi_{M}\right\rangle \rightrightarrows\left\langle N, \chi_{N}\right\rangle$ be two different comodule homomorphisms. Then there exists an element $m \in M$ such that

\footnotetext{
${ }^{2}$ card $(X)$ means the cardinality of the set $X$.
} 
$f(m) \neq g(m)$. Then by Theorem 22 there exists a subcomodule $M^{\prime}$ containing the submodule generated by $m$;

$\langle m\rangle \subset M^{\prime} \subset M$. Furthermore $\operatorname{card}\langle m\rangle \leqq \operatorname{card} k$. Hence card $M^{\prime} \leqq$ $\max \left(\operatorname{card} k, \chi_{0}\right)$ and $f_{i} \neq g_{i}:\left\langle M^{\prime}, \chi_{M^{\prime}}\right\rangle \stackrel{i}{\rightarrow}\left\langle M, \chi_{M}\right\rangle \underset{g}{\stackrel{f}{\rightarrow}}\left\langle N, \chi_{N}\right\rangle$.

ExAmple 25. Let $C=\boldsymbol{Z} \oplus \boldsymbol{Q} / \boldsymbol{Z}$. Then the "set" of denumerable $\boldsymbol{Z} \oplus \boldsymbol{Q} / \boldsymbol{Z}$-comodules generates the category Comod- $\boldsymbol{Z} \boldsymbol{Q} / \boldsymbol{Z}$.

Since Comod- $C$ is cocomplete, cowellpowered and has a generator we obtain by applying the special functor theorem [cf. [13] p. 114 Corollary].

Corollary 26. The category Comod-C is complete. Moreover Comod-C is locally presentable in the sense of Gabriel-Ulmer. ${ }^{3}$

This Corollary shows only the existence of arbitrary limits in Comod- $C$ but gives us no explicit description. Our next step will be therefore to describe explicitly the limits. This was not known even in the case where $k$ is a field. We apply Linton's techniques of constructing colimits in an Eilenberg-Moore category over Sets (cf. [14] Chap. 21)

Construction of limits in Comod-C 27. Let $I$ be a small category and $D: I \rightarrow$ Comod- $C$ be a diagram. Let $(\lim U D, \varphi)$ be the limit of $U D$ in $k-\operatorname{Mod}$ and $(\lim (-\otimes C \cdot U \cdot D, \psi)$ the limit of $-\otimes C U \cdot D$ in $k$-Mod. If $I$ is void then $\lim D$ is the zero comodule. Now let $I$ be nonvoid. Let $\eta: I d_{\text {comod }-C} \rightarrow-\otimes C \cdot U$ be the functorial morphism defined by

$$
\begin{aligned}
& \chi=\eta(\langle M, \chi\rangle):\langle M, \chi\rangle \longrightarrow\langle M \otimes C, M \otimes \Delta\rangle \\
& \begin{array}{c}
M \stackrel{\chi}{\longrightarrow} M \otimes C \\
\chi \downarrow \\
M \otimes C \stackrel{M \otimes x}{\longrightarrow} M \otimes \Delta \\
\stackrel{\downarrow}{\longrightarrow} C \otimes C
\end{array}
\end{aligned}
$$

Then there is exactly one $k$-morphism

$$
\eta^{*}: \lim (U D) \longrightarrow \lim (-\otimes C \cdot U D)
$$

such that the following diagram commutes:

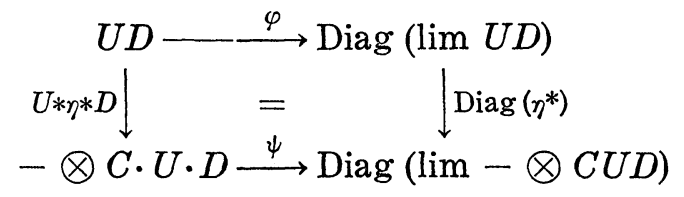

${ }^{3}$ The set of generators in Comod-C is $\boldsymbol{\aleph}_{1}$-presentable-(Ulmer). 
where Diag is the diagonal functor.

Let $\lim U D=M$ and $\lim -\otimes C \cdot U \cdot D=N$. Then there exists exactly one $k$-morphism $\varphi^{*}: M \otimes C \rightarrow N$ such that $-\otimes C * \varphi=$ $\psi \cdot \operatorname{Diag}\left(\varphi^{*}\right)$. We claim that $\eta^{*}$ is a monomorphism. Consider

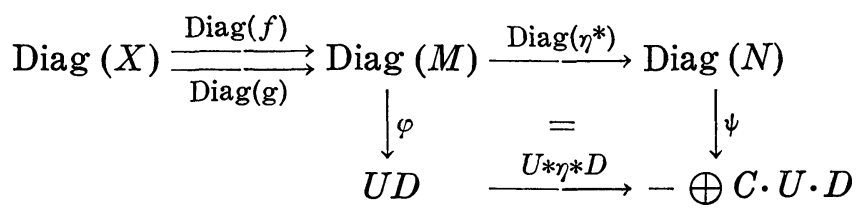

where $f, g: X \rightarrow M$ are $k$-morphisms with $\eta^{*} \cdot f=\eta \cdot g$. Since $(U,-\otimes C)$ is an adjoint functor pair $U * \eta$ is a coretraction and hence also $U * \eta * D$. Thus we obtain $\varphi \operatorname{Diag}(f)=\varphi \operatorname{Diag}(g)$ and hence $f=g$ since $\varphi$ is a universal morphism.

Consider now the cofree comodules $\langle M \otimes C, M \otimes \Delta\rangle$ and $\langle N \otimes C$, $N \otimes \Delta\rangle$ and the comodule homomorphisms

$$
\varphi^{*} \otimes C \cdot M \otimes \Delta, \eta^{*} \otimes C: M \otimes C \longrightarrow N \otimes C \text {. }
$$

Let $\left\langle K, \chi_{K}\right\rangle \stackrel{m}{\rightarrow}\langle M \otimes C, M \otimes \Delta\rangle \overline{\overline{\varphi^{*} \otimes C \cdot M}} \frac{\eta^{*} \otimes C}{\longrightarrow}(N \otimes C, N \otimes \Delta\rangle \quad$ be $\quad$ an equalizer of $\left(\eta^{*} \otimes C, \varphi^{*} \otimes M \otimes \Delta\right)$. Then $\left\langle K, \chi_{K}\right\rangle$ is the limit of $D$ in Comod- $C$.

This is now shown in several steps (cf. [16] 21. 2. 10).

EXAMPLE 28. Let $C$ be a flat coalgebra. Then the finite limits and in particular the equalizers in Comod- $C$ are formed in $k$-Mod. We want now to compute the products in Comod-C. Let $\left\langle M_{i}, \chi_{i}\right\rangle$; $i \in I$, be a family of $C$-comodules. Denote by $\Pi M_{i}$ the product of the underlying $k$-modules and by $\Pi M_{i} \otimes C$ the product of the $k$-modules $M_{i} \otimes C$. Then we obtain two canonical morphisms $\eta^{*}$ and $\varphi^{*}$ defined by the universal property of $\Pi M_{i} \otimes C$ :

$$
\begin{gathered}
M_{i} \otimes C \stackrel{\text { can }}{\longleftarrow} \Pi M_{i} \otimes C \\
\downarrow_{i}=\underset{\downarrow}{=} \Pi \chi_{i}=\eta^{*} \\
M_{i} \stackrel{\text { can }}{\longleftarrow} \Pi M_{i}^{\prime}
\end{gathered}
$$

and

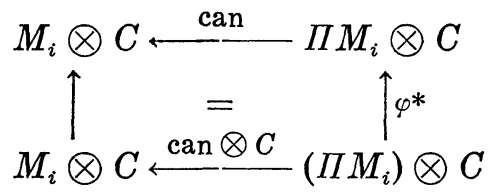

with $\varphi^{*}\left(\left(m_{i}\right) \otimes c\right)=\left(m_{i} \otimes c\right)$ and $\eta^{*}\left(m_{i}\right)=\left(\chi_{i}\left(m_{i}\right)\right)$. Then the equalizer of 


$$
\left(\Pi M_{i}\right) \otimes C \underset{\varphi^{*} \otimes C \cdot\left(\Pi M_{i}\right) \otimes \Delta}{\stackrel{\eta^{*} \otimes C}{\longrightarrow}}\left(\Pi M_{i} \otimes C\right) \otimes C
$$

is the product of the family $\left\langle M_{i}, \chi_{i}\right\rangle$ in Comod- $C$, i.e.,

$$
\begin{aligned}
\prod_{\text {comod }-C}\left\langle M_{i}, \chi_{i}\right\rangle & =\left\{\sum_{\text {finite }} \bar{m}_{k} \otimes C_{k} \in\left(\Pi M_{i}\right) \otimes C ; \sum_{\text {finite }}\left(\chi_{i}\left(m_{i}^{k}\right)\right) \otimes C_{k}\right. \\
& \left.=\sum_{\text {finite }} \sum_{\left(C_{k}\right)}\left(m_{i}^{k} \otimes C_{k(1)}\right) \otimes C_{k(2)}\right\}
\end{aligned}
$$

where $\bar{m}_{k}=\left(m_{i}^{k}\right) i \in I$ and $\Delta C_{k}=\sum_{\left(C_{k}\right)} C_{k(1)} \otimes C_{k(2)}$ with the comodule structure induced by the comodule structure $\left(\Pi M_{i}\right) \otimes \Delta$ and $\left(\Pi M_{i} \otimes\right.$ $\varepsilon\left(\Pi M_{i}\right) \otimes C$. The projections $p_{i}$ are given by the following assignments.

$$
p_{i}: \prod_{\text {cmod- } C}\left\langle M_{i}, \chi_{i}\right\rangle \longrightarrow\left\langle M_{i}, \chi_{i}\right\rangle \sum_{\text {finite }}\left(m_{i}^{k}\right) \otimes C_{k} \longmapsto \varepsilon\left(C_{k}\right) \cdot m_{i}^{k} .
$$

Let us now consider the functorial morphism (functorial in $C$ )

$$
\lambda: k-\operatorname{Mod}(M, N \otimes C) \longrightarrow k-\operatorname{Mod}\left(C^{*} \otimes M, N\right)
$$

defined by $\lambda(f)\left(c^{*} \otimes m\right)=\left(1 \otimes c^{*}\right) f(m)$ where $C^{*}=k$-Mod $(\mathrm{C}, k)$. If $C$ is a coalgebra then $C^{*}$ is a $k$-algebra with the multiplication

$$
f * f^{\prime}(c)=\sum_{(c)} f\left(c_{(1)}\right) \cdot f^{\prime}\left(c_{(2)}\right)
$$

and unit $e(c)=\varepsilon(c)$. (cf. [14]) Let $C$ be a coalgebra and $\langle M, \chi: M \rightarrow$ $M \otimes C>$ a comodule. Then $M$ is a $C^{*}$-left module with multiplication: $\lambda(\chi): C^{*} \otimes M \rightarrow M$. The assignments

$$
\begin{aligned}
\lambda: \text { Comod-C } & \longrightarrow C^{*} \text {-Mod } \\
\langle M, \chi\rangle & \longmapsto\langle M, \lambda(\chi)\rangle \\
f & \longmapsto f
\end{aligned}
$$

define a functor (cf. [14]).

TheOREM 29. $\lambda$ : Comod-C $\rightarrow C^{*}-$ Mod is comonadic. In particular $\lambda$ has a right adjoint.

Proof. Since Comod- $C$ is cocomplete, cowellpowered and has a generator, $\lambda$ has a right-adjoint if and only if $\lambda$ preserves colimits (special adjoint functor theorem). Let

$$
\left\langle M_{i}, \chi_{i}\right\rangle \stackrel{m_{i}}{\longrightarrow}\left\langle\operatorname{colim} M_{i}, \chi\right\rangle
$$

be a colimit diagram in Comod-C. Then $\lambda(\chi): C^{*} \otimes \operatorname{colim} M_{i} \rightarrow$ colim $M_{i}$ is a colimit of $\left\langle M_{i}, \lambda\left(\chi_{i}\right)\right\rangle, i \in I$, as one easily computes. 
Hence $\lambda$ preserves colimits and thus has a right adjoint. Next I'll show that $\lambda$ creates equalizer of $\lambda$-contractible pairs. Let $f, g:\langle A$, $\left.\chi_{A}\right\rangle \rightrightarrows\left\langle B, \chi_{B}\right\rangle$ be a pair of $\lambda$-contractible Comod- $C$ morphisms and $m: K \rightarrow A$ be an equalizer of $f, g:\left\langle A, \lambda\left(\chi_{A}\right)\right\rangle \rightrightarrows\left\langle B, \lambda\left(\chi_{B}\right)\right\rangle$ in $C^{*}$-Mod. Then there exist $C^{*}$-module homomorphisms $h:\left\langle B, \lambda\left(\chi_{B}\right)\right\rangle \rightarrow\left\langle A, \lambda\left(\chi_{A}\right)\right\rangle$ and $k:\left\langle A, \lambda\left(\chi_{A}\right)\right\rangle \rightarrow K$ such that the following diagram commutes:

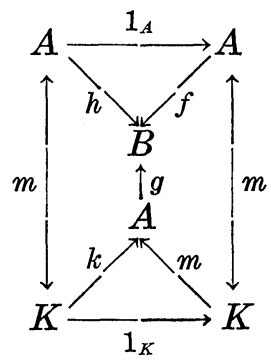

Since functors preserve equalizers of contractible pairs, $K \stackrel{m}{\rightarrow} A \underset{g}{\stackrel{f}{\rightarrow}} B$ is an equalizer of the contractible pair $(f, g)$ in $k$-Mod. Since $U:$ Comod- $C \rightarrow k$-Mod is comonadic, $K$ carries a comodule structure $\chi_{K}$ such that $\left\langle K, \chi_{K}\right\rangle \stackrel{m}{\rightarrow}\left\langle A, \chi_{A}\right\rangle \underset{g}{\stackrel{f}{\rightrightarrows}}\left\langle B, \chi_{B}\right\rangle$ is an equalizer diagram in Comod-C. Hence $\lambda$ creates equalizers of $\lambda$-contractible pairs and hence is comonadic.

REMARKs 30. (1) The fact that $\lambda$ creates equalizers of $\lambda$ contractible pairs follows also from the following:

Lemma. Let $f, g\left\langle A, \chi_{A}\right\rangle \rightrightarrows\left\langle B, \chi_{B}\right\rangle$ be a pair of comodule homomorphisms and $K \stackrel{m}{\rightarrow} A \underset{g}{\stackrel{f}{\rightrightarrows}} B$ the equalizer of $f, g$ in $k$-Mod. If $m$ is a coretraction in $k$-Mod then $K$ carries a comodule structure $\chi_{K}$ such that

$$
\left\langle K, \chi_{K}\right\rangle \stackrel{m}{\longrightarrow}\left\langle A, \chi_{A}\right\rangle \underset{g}{\stackrel{f}{\longrightarrow}}\left\langle B, \chi_{B}\right\rangle
$$

is an equalizer diagram in Comod-C.

Let $m$ be an equalizer of a $\lambda$-contractible pair $f, g$. Then $m$ is a coretraction in $k$-Mod and hence an equalizer in Comod- $C$, i.e., $\lambda$ creates equalizers of $\lambda$-contractible pairs.

(2) The fact that $\lambda$ is comonadic follows immediately from the following Dubuc-triangle 


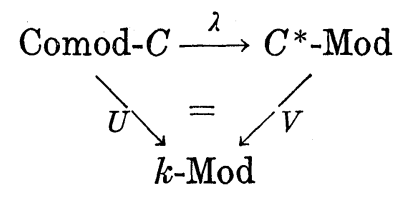

where $U$ and $V$ are the underlying functors. Since $U$ and $V$ are comonadic and Comod- $C$ has equalizer, $\lambda$ is also comonadic (cf. [20] Proposition 6.11).

(3) If $C$ is finite ( $\equiv$ finitely generated and projective) then $\lambda$ : Comod- $C \rightarrow C^{*}$-Mod is an isomorphism of categories (cf. [14]).

The next proposition solves the problem of the existence of free comodules i.e. answers the following question: For which coalgebras $C$ does the forgetful functor $V:$ Comod- $C \rightarrow$ Sets have a left-adjoint?

Proposition 31. The following statements are equivalent: adjoint.

(i) The forgetful functor $V$ : Comod-C $\rightarrow$ Sets has a left-

(ii) $C$ is finite i.e. finitely generated and projective.

(iii) $-\otimes C: k$-Mod $\rightarrow k$-Mod preserves limits.

(iv) $\lambda$ : Comod-C $\rightarrow C^{*}$-Mod has a left-adjoint.

(v) $U$ : Comod-C $\rightarrow k$-Mod preserves limits.

If one of these conditions is fulfilled then $\lambda$ : Comod-C $\rightarrow C^{*}-\operatorname{Mod}$ is an isomorphism.

Proof. The equivalences (i) $\leftrightarrow$ (iii) $\leftrightarrow$ (iv) $\leftrightarrow$ (v) are categorical routine. The equivalence (iii) $\leftrightarrow$ (ii) follows from the well-known fact that $-\otimes C$ preserves limits if and only if $C$ is finitely presented and flat or equivalently if $C$ is finitely generated and prejective. If one of these conditions is fulfilled then $\lambda$ is an isomorphism by (30.3).

Description of the free C-comodules 32 . Let $C$ be a finitely generated and projective coalgebra. The above proposition gives us the following explicit description of the free $C$-comodules: Let $X$ be an arbitrary set. Then the free $C$-comodule $F X$ generated by $X$ is given by $F X \cong \bigoplus_{X} C^{*}$ where $C^{*}$ has the "canonical" $C$-comodule structure.

CoRollary 33. Notation as above. The functor $\lambda$ : Comod-C $\rightarrow$ $C^{*}$-Mod is an isomorphism if and only if $C$ is finitely generated and projective.

Next we consider factorizations in Comod-C. Let us first recall some of the basic notions and propositions (cf. [20]). Let $A$ be a 
category. For two $A$-morphisms $e: A \rightarrow B$ and $m: C \rightarrow D$ we write $e \downarrow m$ if every commutative diagram

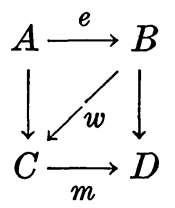

can be made commutative by a unique morphism $w: B \rightarrow C$. Let $P$ be any class of $\boldsymbol{A}$-morphisms. Then $p^{\dagger}$ resp. $p^{\downarrow}$ shall denote the following classes of $\boldsymbol{A}$-morphisms.

$$
\begin{aligned}
& p^{\uparrow}=\{e ; e \downarrow m \text { for all } m \in P\} \\
& p^{\downarrow}=\{m ; e \downarrow m \text { for all } e \in P\} .
\end{aligned}
$$

$A$ pair $(E, M)$ of classes $E$ and $M$ of $A$-morphisms is a prefactrization in $\boldsymbol{A}$ if $E=M^{\uparrow}$ and $M=E^{\downarrow}$. A prefactorization $(E, M)$ is called a factorization in $\boldsymbol{A}$ if every morphism $f$ in $\boldsymbol{A}$ is of the form $f=m \cdot e$ with $m \in M$ and $e \in E$. A factorization $(E, M)$ is proper if every $e \in E$ is an epimorphism and every $m \in M$ is a monomorphism. Hence a proper factorization on $\boldsymbol{A}$ is the same thing as a bicategorical structure in the sense of Isbell. We say that a category $\boldsymbol{A}$ has a $M$-factorization if $\boldsymbol{A}$ has a $\left(M^{\dagger}, M\right)$-factorization. Let $\boldsymbol{K}$ and $\boldsymbol{L}$ be categories with factrizations $M_{K}$ resp. $M_{L}$. A functor $F: \boldsymbol{K} \rightarrow \boldsymbol{L}$ is said top reserve $M_{K}$-factorizations if $F\left(M_{K}\right) \subset M_{L}$ and $F\left(M_{K}^{\uparrow}\right) \subset M_{L}^{\uparrow}$. $F$ is said to reflect $M_{L}$-factorizations if $F^{-1}\left(M_{L}\right) M_{K}$ and $F^{-1}\left(M_{L}^{\dagger}\right) \subset M_{K}^{\dagger}$. Let $H_{\boldsymbol{K}} \subset$ Mor $\boldsymbol{K}$ with Iso $(\boldsymbol{K}) \subset H_{\boldsymbol{X}}$ and $H_{\boldsymbol{K}}$ Iso $(\boldsymbol{K}) \subset H_{\boldsymbol{K}}$. A functor $F: \boldsymbol{K} \rightarrow \boldsymbol{L}$ is said to create $H_{K}$-factorizations from $M_{\boldsymbol{L}}$-factorizations if for all $f \in$ Mor $\boldsymbol{K}$ with

$$
F f=m_{L} e_{L}, m_{L} \in M_{L}, e_{L} \in M_{L}^{\uparrow}
$$

there is a unique factorization $f=m_{K} \cdot e_{K}$ in $\boldsymbol{K}$ with $F_{m K}=m_{L}$, $F e_{K}=e_{L}, m_{K} \in H_{K}, e_{K} \in{ }_{K}^{\uparrow}$.

Proposition 34. Let $\boldsymbol{K}$ be a cocomplete, cowellpowered category. Then $\boldsymbol{K}$ has an (epi, extremal mono)-factorization i.e., a factorization $(E, M)$ where $E$ is the class of all epimorphisms and $M$ is the class of all extremal monomorphisms (Isbell-Kennison).

Hence the category Comod- $C$ has at least one proper factorization.

Proposition 35. Let $(E, M)$ be a proper factorization in Comod-C. Then the following statement are equivalent.

(i) The underlying functor $U$ : Comod-C $\rightarrow k$-Mod preserves the 
factorization.

(ii) $U$ is exact.

(iii) $C$ is flat.

Proof. Since (ii) and (iii) are equivalent by Proposition 8 and since the implication (iii) $\rightarrow$ (i) is trivial we have only to prove (i) $\rightarrow$ (iii). Let $E_{k}$ resp. $M_{k}$ be the class of all epimorphisms resp. monomorphisms in $k$-Mod. Since $U$ preserves the factorization and $U$ reflects isomorphisms we obtain that $E=U^{-1}\left(E_{k}\right)$ and $M=U^{-1}\left(M_{k}\right)$. Since $U(E) \subset E_{k}$ and $-\otimes C$ is right adjoint to $U$ we get $\left(M_{k}\right) \otimes C \subset M$. Hence we get for the functor $-\otimes C: k-\operatorname{Mod} \rightarrow k$-Mod

$$
\left(M_{k}\right) \otimes C=U(-\otimes C)\left(M_{k}\right) \subset(M) \subset M_{k}
$$

i.e., $-\otimes C$ preserves monomorphisms.

COROLLARY 36. The underlying functor $U$ : Comod-C $\rightarrow k$-Mod creates factorizations from $E_{k}$-factorizations in $k$-Mod if and only if $C$ is flat.

Proposition 35 shows that, if $C$ is not flat, then an arbitrary $C$-comodule homomorphism can not be factorized through a surjective comodule homomorphism and an injective comodule homomorphism. In particular the canonical (epi-mono)-factorization of a comodule homomorphism in $k$-Mod cannot be lifted to a factorization in Comod-C. In the sequel $(E, M)$ shall always denote the proper factorization (epi, extremal mono) on Comod-C. Words as epimorphism, monomorphism, generator, wellpowered $\cdots$ are used in a sense relative to $(E, M)$.

Proposition 37. Comod-C is wellpowered relative to the factorization (epi, extremal mono). ${ }^{4}$

Proof. In the same vein as the proof for Proposition 10.6.3 in $[16]$.

For the rest of this paper we will use the property that the category $k$-Mod is a symmetrical monoidal closed category with respect to the tensor product, and that Comod- $C$ is an enriched category over $k$-Mod. In the following we will study the left adjoints of the $k$-Mod-representable functors called tensors and cotensors. They provide a characterisation of certain constructions which is not available in an ordinary set based approach. Cotensors will play an important role in duality theory (i.e. Gelfand theory)

\footnotetext{
${ }^{4}$ Comod- $C$ is even wellpowered with respect to all monos.
} 
as it will be shown in part II of the present work. We use the language in [6].

Comod- $C$ is a $k$-Mod-category. The internal Hom-functor [, ]: Comod- $C^{\text {op }} \times$ Comod- $C \rightarrow k$-Mod is gived by $[M, N]=$ Comod- $C(M, N)$. The pair of adjoint functors Comod-C $\rightleftarrows k$-Mod is a pair of $k$-Modfunctors. In the sequel we call $k$-Mod-functors $k$-linear functors.

Proposition 38. The category Comod-C is tensored i.e. for every $k$-module $M$ and every $C$-comodule $X$ the functor Comod- $C \rightarrow$ $k$-Mod: $Y \mapsto k-\operatorname{Mod}(M$, Comod- $C(X, Y))$ is representable over $k$-Mod.

Proof. Let $M \in k$-Mod and $X \in$ Comod-C. The $M \otimes X$ is a $C$ comodule. The rest follows from the canonical $k$-linear isomorphism $\operatorname{Comod}-C(M \otimes X, Y) \cong k-\operatorname{Mod}(M, \operatorname{Comod}-C(X, Y))$.

CoRollary 39. The cofree k-linear functor $-\otimes C: k$-Mod $\rightarrow$ Comod-C has a k-linear right adjoint functor represented by the $k$ linear functor Comod- $C(C,-)$.

Proposition 40. The category Comod- $C$ is cotensored i.e. for every $M \in k$-Mod and $X \in$ Comod- $C$ the functor Comod- $C^{\circ p} \rightarrow k$-Mod: $Y \mapsto k-\operatorname{Mod}(M$, Comod $(Y, X))$ is representable.

Proof. Since Comod- $C$ is a tensored category Comod- $C$ is cotensored if and only if for every $k$-module $M$ the $k$-linear functor $F_{M}: M \otimes-$ : Comod- $C \rightarrow$ Comod- $C$ has a $k$-linear right adjoint. Let $N \otimes X$ be a tensor with $N \in k$-Mod and $X \in$ Comod- $C$ as above. Then $F_{M}(N \otimes X)=M \otimes(N \otimes X) \cong N \otimes(M \otimes X) \cong N \otimes F_{M}(X)$. Hence $F_{M}$ is a tensor preserving functor in the sense of [6]. Since $F_{M}$ preserves colimits, $F_{M}$ has a right adjoint by the Special Adjoint Functor Theorem. Since $F_{M}$ preserves tensors the right adjoint $\overline{\text { Comod-C }}(M,-)$ is a $k$-linear functor and the representation Comod- $C$ $(X, \overline{\operatorname{Comod}-C}(M, X)) \cong \operatorname{Comod}-C(M \otimes X, Y) \cong k-\operatorname{Mod}(M, \operatorname{Comod}(X, Y))$ is $k$-linear.

Corollary 41. Comod-C is k-Mod-complete and k-Mod-cocomplete.

Let $f: C \rightarrow C^{\prime}$ be a coalgebra morphism. Then $f$ induceds a functor $f^{*}$ : Comod- $C \rightarrow$ Comod- $C^{\prime}$ by the assignment $\left(M, \chi_{M}\right) \mapsto$ $\left(M, 1 \otimes f \chi_{M}\right)$. Then $f^{*}$ is obviously a $k$-linear functor. By [15] 21.2.1 the mapping $f \mapsto f^{*}$ induces a bijection between Coalg $\left(C, C^{\prime}\right)$ and the "set" of all functors $\varphi:$ Comod-C $\rightarrow$ Comod-C" with $U_{C}=U_{C}, \varphi$.

Proposition 42. Let $f: C \rightarrow C^{\prime}$ be a coalgebra morphism. Then 
(1) $f^{*}$ preserves tensors.

(2) $f^{*}$ has a k-linear right adjoint $f_{*}$.

Proof. The assertion 1 is trivial. Since $f^{*}$ preserves colimits it has a right adjoint by the Special Adjoint Functor Theorem. Since $f^{*}$ preserves tensors the right adjoint is $k$-linear.

Description of the functor $f_{*} 43$. Let $M$ be a $C$-right comodule and $N$ a $C$-left comodule. The tensor coproduct of $M$ and $N$ under $C$ denoted by $M \otimes^{C} N$ is given by the following equalizer digram in $k$-Mod.

$$
M \otimes c N \longrightarrow M \otimes N \underset{M \otimes \chi_{N}}{\stackrel{\chi_{M} \otimes M}{\longrightarrow}} M \otimes C \otimes N
$$

Then if $f: C \rightarrow C^{\prime}$ is a coalgebra morphism between flat coalgebras $C$ and $C^{\prime}$ the functor $f_{*}$ : Comod- $C^{\prime} \rightarrow$ Comod- $C$ is given by the following assignment $f_{*}\left(M, \chi_{M}\right)=\left(M \otimes^{C} C, 1_{M} \otimes^{C} \Delta\right)$.

Final Observation 44. In the same vein as I studied the category of comodules for a fixed coalgebra one can study the category Comod of all comodules i.e. pairs $\left(\left(M, \chi_{M}\right), C\right)$ where $\left(M, \chi_{M}\right)$ is a comodule over $C$. One obtains similar results. The starting point for the study of this category is the following theorem

THEOREM 45. The underlying functor

$$
U: \text { Comod } \longrightarrow k-\operatorname{Mod} \times k \text {-Coalg: }\left(\left(M, \chi_{M}\right), C\right) \longmapsto(M, C)
$$

is comonadic.

This note was written during my visit to the University of California at San Diego. I would like to thank in particular Professor Helmut Röhrl for his hospitality and the stimulating discussions on this paper. Furthermore I am indebted to Professor Bodo Pareigis for stimulating the study of comodules over an arbitrary coalgebra.

\section{REFERENCES}

1. M. Alderman, Abelian categories over additive ones, J. of Pure and Applied Algebra

3, (1973), 103-117.

2. F. Anderson, K. Fuller, Rings and Categories of Modules, Springer, New York, Heidelberg, Berlin, 1974.

3. M. Barr, Coalgebras over arbitrary commutative rings, To appear in J. of Algebra.

4. N. Bourbaki, Algèbre commutative $I$ \& 2, Hermann, Paris, 1961.

5. M. Demazure, P. Gabriel, Groupes algébriques, Tome I. North Holland Co. Amsterdam 1970. 
6. E. J. Dubuc, Kan Extensions in Enriched Category Theory LN 145, Springer, Berlin, Heidelberg, New York, 1970.

7. S. Eilenberg, T. C. Moore, Foundations of relative homological algebra, Amer. Math. Soc., 1965.

8. R. Faber, P. Freyd, Fill-in Theorems, in: Proc. Conf. on Categorical Algebra, La Jolla, Springer, Berlin, 1966.

9. P. Freyd, Representations in Abelian Categories, in: Proc. Conf. on Categorical Algebra, La Jolla, 1965 Springer, Berlin, 1966.

10. D. W. Jonah, Cohomology of coalgebras, Amer. Math. Soc., 1968.

11. S. Maclane, Categories for the Working Mathematician, Springer, New York, Heidelberg, Berlin. 1973.

12. B. Mitchell, Theory of Categories, Academic Press, New York, London, 1965.

13. B. Pareigis, Categories and Functors, Academic Press, New York, London, 1970.

14. B. Pareigis, Endliche Hopfalgebren, Vorlesungsausarbeitung, München, 1973.

15. R. Saavedra, Catégories Tannakiennes, Springer, New York, Heidelberg, Berlin, LN 265, 1972.

16. H. Schubert, Categories, Springer, New York, Heidelberg, Berlin, 1972.

17. Séminaire "SOPHUS LIE"/1955/56. Hyperalgèbres et groupes de Lie formels, Paris, 1957.

18. W. Settele, Über die Eigenschaften der Kategorie der Comoduln über eimer Coalgebra, Diplomarbeit, München, 1974.

19. Sweedler. Hopfalgebras, Benjamin, New York, 1969.

20. W. Tholen, Relative Bildzerlegungen und algebraische Kategorien, Dissertation, Münster, 1975.

21. T. Wuerfel, Ueber absolut reine Ringe, Dissertation, München, 1971.

Received May 16, 1975.

University of California, San Diego

AND

Mathematisches Institut DeR UNIVersität MÜNCheN

Current Address: Fachbereich Mathematik der Universität Bremen 


\section{PACIFIC JOURNAL OF MATHEMATICS}

\section{EDITORS}

RICHARD ARENS (Managing Editor)

University of California

Los Angeles, California 90024

\author{
R. A. Beaumont \\ University of Washington \\ Seattle, Washington 98105
}

\section{J. DugundjI}

Department of Mathematics University of Southern California Los Angeles, California 90007

D. Gilbarg and J. Milgram

Stanford University

Stanford, California 94305

\section{ASSOCIATE EDITORS}
E. F. BECKENBACH
B. H. NeumanN
F. WOLF
K. YoshidA

\section{SUPPORTING INSTITUTIONS}

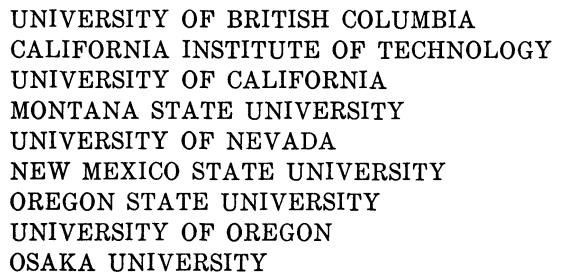

UNIVERSITY OF BRITISH COLUMBIA CALIFORNIA INSTITUTE OF TECHNOLOGY UNIVERSITY OF CALIFORNIA MONTANA STATE UNIVERSITY UNIVERSITY OF NEVADA NEW MEXICO STATE UNIVERSITY OSAKA UNIVERSITY OREGON STATE UNIVERSITY UNIVERSITY OF OREGON

\author{
UNIVERSITY OF SOUTHERN CALIFORNIA \\ STANFORD UNIVERSITY \\ UNIVERSITY OF TOKYO \\ UNIVERSITY OF UTAH \\ WASHINGTON STATE UNIVERSITY \\ UNIVERSITY OF WASHINGTON \\ AMERICAN MATHEMATICAL SOCIETY
}

The Supporting Institutions listed above contribute to the cost of publication of this Journal, but they are not owners or publishers and have no responsibility for its content or policies.

Mathematical papers intended for publication in the Pacific Journal of Mathematics should be in typed form or offset-reproduced, (not dittoed), double spaced with large margins. Please do not use built up fractions in the text of your manuscript. You may however, use them in the displayed equations. Underline Greek letters in red, German in green, and script in blue. The first paragraph or two must be capable of being used separately as a synopsis of the entire paper. Items of the bibliography should not be cited there unless absolutely necessary, in which case they must be identified by author and Journal, rather than by item number. Manuscripts, in triplicate, may be sent to any one of the editors. Please classify according to the scheme of Math. Reviews, Index to Vol. 39. All other communications should be addressed to the managing editor, or Elaine Barth, University of California, Los Angeles, California, 90024.

The Pacific Journal of Mathematics expects the author's institution to pay page charges, and reserves the right to delay publication for nonpayment of charges in case of financial emergency.

100 reprints are provided free for each article, only if page charges have been substantially paid. Additional copies may be obtained at cost in multiples of 50 .

The Pacific Journal of Mathematics is issued monthly as of January 1966. Regular subscription rate: $\$ 72.00$ a year $(6$ Vols., 12 issues). Special rate: $\$ 36.00$ a year to individual members of supporting institutions.

Subscriptions, orders for back numbers, and changes of address should be sent to Pacific Journal of Mathematics, 103 Highland Boulevard, Berkeley, California, 94708.

PUBLISHED BY PACIFIC JOURNAL OF MATHEMATICS, A NON-PROFIT CORPORATION

Printed at Kokusai Bunken Insatsusha (International Academic Printing Co., Ltd.), 8-8, 3-chome, Takadanobaba, Shinjuku-ku, Tokyo 160, Japan. 


\section{Pacific Journal of Mathematics}

\section{Vol. 61, No. 2 December, 1975}

Graham Donald Allen, Francis Joseph Narcowich and James Patrick Williams, An operator version of a theorem of Kolmogorov .......................

Joel Hilary Anderson and Ciprian Foias, Properties which normal operators share with normal derivations and related operators . . . . . . . . . . . . . . . . . . . . .

Constantin Gelu Apostol and Norberto Salinas, Nilpotent approximations and

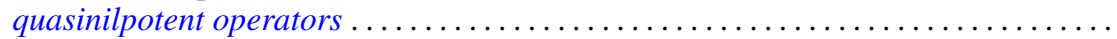

James M. Briggs, Jr., Finitely generated ideals in regular $F$-algebras . . . . . . . . . . .

Frank Benjamin Cannonito and Ronald Wallace Gatterdam, The word problem and power problem in 1-relator groups are primitive recursive ..................

Clifton Earle Corzatt, Permutation polynomials over the rational numbers ...........

L. S. Dube, An inversion of the $S_{2}$ transform for generalized functions . . . . . . . . . . William Richard Emerson, Averaging strongly subadditive set functions in unimodular

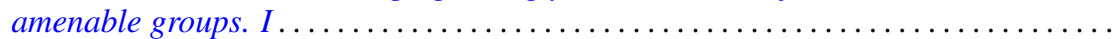

Barry J. Gardner, Semi-simple radical classes of algebras and attainability of

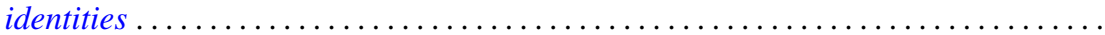

Irving Leonard Glicksberg, Removable discontinuities of A-holomorphic functions ....

Fred Halpern, Transfer theorems for topological structures . . . . . . . . . . . . . . .

H. B. Hamilton, T. E. Nordahl and Takayuki Tamura, Commutative cancellative

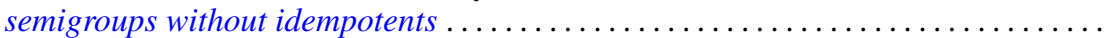

Melvin Hochster, An obstruction to lifting cyclic modules .....................

Alistair H. Lachlan, Theories with a finite number of models in an uncountable power

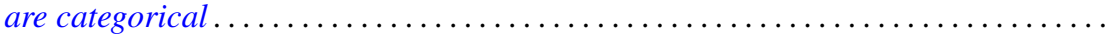

Kjeld Laursen, Continuity of linear maps from $C^{*}$-algebras . . . . . . . . . . . . .

Tsai Sheng Liu, Oscillation of even order differential equations with deviating arguments ....

Jorge Martinez, Doubling chains, singular elements and hyper- $Z$

Mehdi Radjabalipour and Heydar Radjavi, On the geometry of num Thomas I. Seidman, The solution of singular equations, I. Linear equations in Hilbert

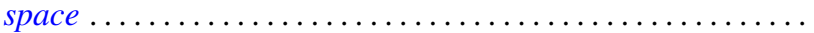

R. James Tomkins, Properties of martingale-like sequences ......

Alfons Van Daele, A Radon Nikodým theorem for weights on von Neumann

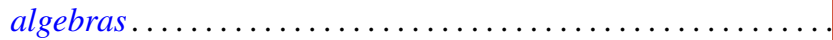

Kenneth S. Williams, On Euler's criterion for quintic nonresidues

Scott Andrew Wolpert, Noncompleteness of the Weil-Petersson metric for Teichmüller

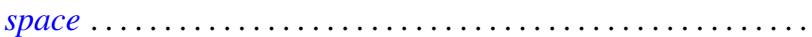

Volker Wrobel, Some generalizations of Schauder's theorem in locally convex

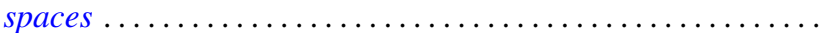

Kelly Denis McKennon, Corrections to: "Multipliers of type $(p, p)$ "; "Multipliers of type $(p, p)$ and multipliers of the group $L_{p}$-algebras"; "Multipliers and the

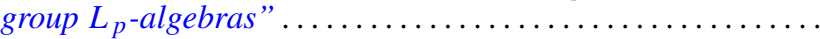

Andrew M. W. Glass, W. Charles (Wilbur) Holland Jr. and Stephen H. McCleary, Correction to: " $a *$-closures to completely distributive lattice-ordered

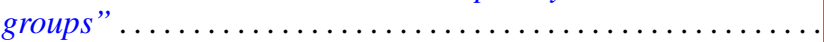

Zvi Arad and George Isaac Glauberman, Correction to: "A characteristic subgroup of

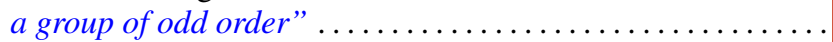

Roger W. Barnard and John Lawson Lewis, Correction to: "Subordination theorems

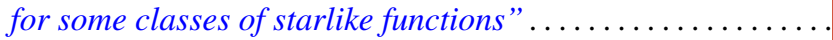

